\title{
Chemoreception by a Cell with Age-Dependent Receptor Binding Sites
}

\author{
BERNARD J. GEURTS AND FREDERIK W. WIEGEL \\ Center for Theoretical Physics, Enschede 7500 AE, The Netherlands
}

Received 30 March 1989; revised 21 August 1989

\begin{abstract}
The authors determine the time-dependent ligand current into a spherical cell that is covered with a large number of age-dependent receptors. These receptors can be in either of two states: active (i.e., available for ligand binding) or inactive. An active receptor turns inactive upon binding a ligand, and it can reappear as active at some later time. The transition inactive $\rightarrow$ active is treated as a probabilistic process. The ligand distribution around the cell is determined analytically in terms of this distribution at the cell surface. A set of nonlinear integral equations is derived for the distribution at the cell surface, which is solved numerically. In this way the time-dependent ligand current into the cell as well as the average active receptor population at the cell surface are determined.
\end{abstract}

\section{INTRODUCTION}

In a previous paper [7] we started to apply the boundary condition approach of DeLisi and Wiegel [4] to the problem of chemoreception by a cell with nonideal receptors. We assumed that the receptors become permanently blocked after binding ligands. In this paper we extend the treatment to cover cells with more general nonideal receptors. These receptors can be in either of two states: active or inactive. An active receptor is available for ligand binding and turns inactive upon binding ligands. An inactive receptor can subsequently turn active again with a probability per unit of time that is assumed to depend on the time elapsed since that receptor last became inactive by binding ligands. The ligand current into the cell and the average active receptor population at the cell surface are determined analytically. Several numerical illustrations are given.

Most treatments of chemoreception deal with a single cell that carries a large number, $O\left(10^{4}\right)$, of ideal receptors at its outer membrane specific for certain ligands in the exterior medium. Once such an ideal receptor has bound a ligand, it is assumed to be instantansously available for new ligand binding. One may, however, expect that a certain amount of time is required 
before a receptor becomes available for further ligand sinding after having just bound a ligand. Hence, the total receptor population consists of two groups: an "active" group that is available for ligand binding and an "inactive" group that consists of receptors that at some previous time bound a ligand and have not yet reappeared as active. In this paper we model the reappearance of such inactive receptors as a probabilistic process. An inactive receptor can reappear as active with a probability per unit of time that depends on its "age"-the time elapsed since the last time a ligand was bound by that receptor.

We formulate the mathematical model in Section 2 and derive the solution for the ligand distribution around the cell. This solution is expressed in terms of the solution at the cell surface. In Section 3 a set of nonlinear integral equations for this ligand distribution at the cell surface is derived and solved numerically. We give some exaruples of the time-dependent ligand current into the cell and the average active receptor population for a specific "waiting-time" distribution in Section 4 and derive the limiting values for these quantities in the long time regime.

\section{FORMULATION OF THE MODEL GOVERNING THE LIGAND DISTRIBUTION}

Consider a spherical cell of radius $\boldsymbol{R}$ placed _. an infinite medium that initially contains ligands at uniform concentration. In the cell membrane $N_{0} \gg 1$ randomly distributed receptors, each of which is specinic ior the ligands in the exterior medium, are immersed. It is assumed that ligands are bound instantaneously to active receptors and get transferred to the interior of the cell. The receptors are assumed to be characterized by a single linear dimension $s[8]$ and a return probability density $\rho$. This return probability density characterizes the process in $\boldsymbol{r}$ 'ich inactive receptors reappear as active and is assumed to be a function of the "age" of the inactive receptor only, that is, of the time elapsed since the receptor last became inactive by binding ligands. The probability that an inactive receptor that became inactive at $t=0$, say, will reappear as active between $t$ and $t+d t$ is thus given by $\rho(t) d t$. This probability density determines the dynamics of the average active receptor population and hence the ligand distribution around the ceili. The following treatment is valid for arbitrary $\rho$. However, one may expect that $\rho$ will be peaked around some value of its argument, characterizing the fact that most inactive receptors reappear as active roughly after the same amount of time elapsed since the last time that receptor became inactive. This case will be treated explicitly in Section 4.

Let $c(r, t)$ denote the number density of ligands at time $t$ and position $r$. As was shown before [8], the evolution of $c(r, t)$ is governed by the diffusion 
equation, which in view of the spherical symmetry of the problem reads

$$
\frac{1}{D} \partial_{t} c(r, t)=\partial_{r r} c(r, t)+\frac{2}{r} \partial_{r} c(r, t),
$$

in which $r=|r|>R$ and $t>0$. D is the diffusion coefficient that characterizes the translational motion of the ligands in the exterior medium. As the initial condition we take the homogeneous situation

$$
c(r, 0)=c_{0},
$$

where $c_{0}$ is a constant ligand concentration. As was shown in [7] and [8], the boundary condition at the cell surface can be written as

$$
c(R, t)-\alpha(t) \partial_{r} c(R, t)=0,
$$

where

$$
\alpha(t)=\frac{\pi R^{2}}{s N(t)}
$$

Here $N(t)$ is the average number of active receptors at time $t$. The number of active receptors at the cell's membrane varies continuously due to the binding of ligands by active receptors, which as a result turn inactive, and the reappearance of inactive receptors as active. The return probability density $\rho$ introduced above plays an essential role in the time dependence of $N(t)$, and hence, through the boundary condition (2.3), (2.4) also in the evolution of $c(r, t)$. We turn to the dynamics of the active receptor population in the next section, showing explicitly in what way $\rho$ determines the evolution of the ligand distribution. The boundary condition (2.3), (2.4) expresses the fact that not every encounter of a ligand with the cell leads to binding of the ligand and was the basis for the work reported in [4]-[8]. It results from an asymptotic flux-matching condition $[4,5]$.

We next transform the variables into a dimensionless form and relate the problem posed by $(2.1)=(2.4)$ to a one-dimensional problem. Let

$$
x=\frac{r}{R} ; \quad T=\frac{D}{R^{2}} t ; \quad \frac{c}{c_{0}}=1-\frac{1}{x} f .
$$

We can easily verify that the function $f(x, T)$ satisfies

$$
\begin{gathered}
\partial_{T} f(x, T)=\partial_{x x} f(x, T), \\
f(x, 0)=0, \\
-\partial_{x} f(1, T)+h f(1, T)=h \varphi(T),
\end{gathered}
$$


where we have set

$$
h=\frac{R+\alpha(0)}{\alpha(0)}
$$

and

$$
\varphi(T)=\frac{R}{h \alpha(0)}+\frac{R}{h} \beta(T)[1-f(1, T)]
$$

We used the notation

$$
\begin{gathered}
\frac{1}{\alpha(T)}=\frac{1}{\alpha(0)}+\beta(T), \\
\beta(T)=\frac{s}{\pi R^{2}} \int_{0}^{T} \frac{d N\left(T^{\prime}\right)}{d T} d T^{\prime}
\end{gathered}
$$

The problem posed by (2.6)-(2.8) is strictly one-dimensional and has an analytic solution that can be found, for example, in Carslaw and Jaeger [2]. It was given explicitly in [7] and can be expressed as

$$
\begin{aligned}
f(x, T)= & \frac{R}{h \alpha(0)}\left[1-\operatorname{erf}\left(\frac{x-1}{2 \sqrt{T}}\right)-e^{h[(x-1)+h T]} \operatorname{erfc}\left(\frac{x-1}{2 \sqrt{T}}+h \sqrt{T}\right)\right. \\
& \left.+\frac{2}{\sqrt{\pi}} \int_{0}^{\infty} e^{-\xi} d \xi \int_{0}^{\infty} e^{-\nu^{2}}\left\{e^{-y_{2}} \Omega\left(y_{1}\right)\left[1-f\left(1, y_{1}\right)\right]\right\} d \nu\right],
\end{aligned}
$$

where

$$
\begin{aligned}
& y_{1}=T\left[1-\frac{\left[(x-1)+h^{-1} \xi\right]^{2}}{\left\{2 \sqrt{T}+\left[(x-1)+h^{-1} \xi\right]\right\}^{2}}\right], \\
& y_{2}=\frac{(x-1)+h^{-1} \xi}{\sqrt{T}}\left[\nu+\frac{(x-1)+h^{-1} \xi}{4 \sqrt{T}}\right],
\end{aligned}
$$

and

$$
\Omega(z)=\alpha(0) \beta(z)=\frac{1}{N_{0}} \int_{0}^{z} \frac{d N\left(T^{\prime}\right)}{d T} d T^{\prime}
$$

In this, erf and erfc are the error function and complementary error function, respectively [1]. The solution (2.13) expresses $f(x, T)$ in terms of the solution at the cell surface integrated over all history. In addition, the dynamics of the active receptors is included through the function $\Omega$. We return to this point in the next section, where we relate $\Omega$ to the solution at 
the cell surface as well, thus obtaining a set of simultaneous integral equations for $\Omega(T)$ and $f(1, T)$ that are to be solved numerically.

The quantity of main interest is the ligand current into the cell, $J(t)$, which is defined by

$$
J(t)=D \int_{S} \partial_{r} c(R, t) d S=4 \pi R^{2} D \partial_{r} c(R, t)
$$

where $S$ is the surface of the cell. In view of the transformations (2.5) and the definition of $\Omega$ in (2.16) one has

$$
\frac{J(T)}{J_{0}}=\frac{R}{\alpha(0)}\{1-f(1, T)\}\{1+\Omega(T)\}
$$

where $J_{0}$ is the stationary state ligand current into a perfectly absorbing sphere $\left(\alpha=0\right.$ ). It is given by $J_{0}=4 \pi R D c_{0}$ (see, e.g., [6]). Notice in addition that the fraction of active receptors is given by

$$
\frac{N(T)}{N_{0}}=1+\Omega(T)
$$

if, for convenience we put $N(0)=N_{0}$. We will mainly be concerned with these two quantities in the examples to be presented in Section 4.

\section{A SYSTEM OF NONLINEAR INTEGRAL EQUATIONS FOR THE: SOLUTION AT THE CELL SURFACE}

In this section we study the dynamics of the active receptors, which will result in an expression for $\Omega$ at time $t$ in terms of $\Omega$ at previous times and the solution $f$ at the cell surface. Combination with the solution (2.13) evaluated at $x=1$ yields a system of nonlinear integral equations for $\Omega(T)$ and $f(1, T)$. An iterative approach to these equations will be formulated that furnishes a rapidly converging algorithm from which $\Omega(T)$ and $f(1, T)$ can be determined numerically.

We defined $N(t)$ as the average number of active receptors at time $t$. In addition, we let $n(t)$ dencte the number of inactive receptors at time $t$, so

$$
N(t)+n(t)=N_{0}
$$

For convenience we take $N(0)=N_{0}$. The collection of inactive receptors at time $t$ consists of inactive receptors of various ages. Let $\Psi(t, \tau) d \tau$ be the number of inactive receptors at time $t$ that became inactive between $\tau$ and $\tau+d \tau$. Hence, assuming the stochastic process governing the age-dependent 
distribution of the ieceptors to be stationary, we have

$$
n(t)=\int_{0}^{t} \Psi(t, \tau) d \tau
$$

The number of active receptors $N(t)$ changes with time because (1) active receptors bind ligands and become inactive at ime $t$ and (2) inactive receptors that became inactive at time $\tau<t$ can reappear as active at the present time $t$ with a probability $\rho(t-\tau) d t$. At a given time $t^{\prime}$ the number of active receptors that turned inactive is proportional to the ligand current $J(t) d t^{\prime}$ with a proportionality constant $\kappa$. If $\kappa=1$; an active receptor can bind exactly one ligand before turning inactive. A value of $x$ between 0 and 1 corresponds to situations in which a receptor can bind a certain amount $(1 / x)$ of ligands before turning inactive. Since by assumption the return probability density $\rho$ depends only on the age of the inactive receptors, we have

$$
\frac{d N(t)}{d t}=-\kappa J(t)+\int_{0}^{t} \kappa J(\tau) \rho(t-\tau) d \tau
$$

and, by definition,

$$
\partial, \Psi(t, \tau)=-\kappa J(\tau) \rho(t-\tau)
$$

So

$$
\begin{aligned}
\Psi(t, \tau) & =\kappa J(\tau)\left[1-\int_{\tau}^{t} \rho\left(\tau^{\prime}-\tau\right) d \tau^{\prime}\right] \\
& =\kappa J(\tau) \int_{t-\tau}^{\infty} \rho(y) d y,
\end{aligned}
$$

where we put $y=\tau^{\prime}-\tau$. Notice the preservation of the total number of receptors by differentiating (3.2) with respect to $t$ and using (3.4), showing that $d N / d t=-d n / d t$.

Recalling the expression (2.18) for $J(T)$ and the definition of $\Omega$ in (2.16), we find after some manipulation

$$
\begin{aligned}
\Omega(T)=\Gamma \int_{0}^{T}[- & {\left[1-f\left(1, T^{\prime}\right)\right]\left[1+\Omega\left(T^{\prime}\right)\right] } \\
& \left.+\int_{0}^{T}\left[1-f\left(1, T^{\prime \prime}\right)\right]\left[1+\Omega\left(T^{\prime \prime}\right)\right] \tilde{\rho}\left(T^{\prime}-T^{\prime \prime}\right) d T^{\prime \prime}\right] d T^{\prime}
\end{aligned}
$$


where

$$
\Gamma \equiv 4 \kappa c_{0} s R^{2}
$$

and

$$
\tilde{\rho}(\xi) \equiv \frac{R^{2}}{D} \rho\left(\frac{R^{2}}{D} \xi\right)
$$

The solution at the cell surface $f(1, T)$ obeys (2.13) evaluated at $x=1$, so

$$
\begin{aligned}
f(1, T)=\frac{R}{h \alpha(0)} & {\left[1-e^{-h^{2} T} \operatorname{erfc}(h \sqrt{T})+\frac{2}{\sqrt{\pi}} \int_{0}^{\infty} d \xi e^{-\xi} \int_{0}^{\infty} d v e^{-v^{2}}\right.} \\
& \times\left\{\exp \left[-\frac{h^{-1} \xi}{\sqrt{T}}\left(\nu+\frac{h^{-1} \xi}{4 \sqrt{T}}\right)\right] \Omega\left[T\left(1-\frac{h^{-2} \xi^{2}}{\left(2 v \sqrt{T}+h^{-1} \xi\right)^{2}}\right)\right]\right\} \\
& \left.\times\left\{1-f\left[1, T\left(1-\frac{h^{-2} \xi^{2}}{\left(2 v \sqrt{T}+h^{-1} \xi\right)^{2}}\right)\right]\right\}\right]
\end{aligned}
$$

Equations (3.6) and (3.9) completely specify $\Omega(T)$ and $f(1, T)$ and hence all other quantities of interest. It seems not possible to find an analytical solution for $\Omega(T)$ and $f(1, T)$ in the general case. We define an iterative treatment.

In shorthand notation we may write (3.6) and (3.9) as

$$
\begin{gathered}
\Omega(T)=\Gamma I_{1}(\Omega, f)(T), \\
f(1, T)=\frac{R}{h \alpha(0)}\left[1-e^{\left.-h^{2} T_{e f f}(h \sqrt{T})+\frac{2}{\sqrt{\pi}} I_{2}(\Omega, f)(T)\right],}\right.
\end{gathered}
$$

where $I_{1}, I_{2}$ are operators acting on a pair of functions $(\Omega, f)$ and are defined through the integrals in (3.6) and (3.9), respectively. We define a sequence of functions $\left(\Omega_{n}, f_{n}\right)$ by

$$
\begin{gathered}
\Omega_{n}(T)=\Gamma_{1}\left(\Omega_{n-1}, f_{n-1}\right)(T), \\
f_{n}(1, T)=\frac{R}{h \alpha(0)}\left[1-e^{h^{2} T} \operatorname{erfc}(h \sqrt{T})+\frac{2}{\sqrt{\pi}} I_{2}\left(\Omega_{n-1}, f_{n-1}\right)(T)\right],
\end{gathered}
$$

with $\left(\Omega_{0}, f_{0}\right)=(0,0)$. The sequence $\left(\Omega_{n}, f_{n}\right)$ was found numerically to converge in the maximum norm to $(\Omega, f)$ as $n \rightarrow \infty$ [7]. The integrations involved in (3.12) and (3.13) were performed using Gauss-Laguerre integration [3]. 
The next section is devoted to some examples of a numerical treatment of this iteration scheme and the limiting values of $N(T) / N_{0}$ and $J(T) / J_{0}$ as $T \rightarrow \infty$.

\section{TIME-DEPENDENT LIGAND CURRENT AND RECEPTOR POPULATION FOR A SPECIAL RETURN PROBABILITY DENSITY}

We consider some numerical examples of the chemoreception by a cell with stochastically gating receptors. In particular we want to study the effect of a delay time between the moment at which an active receptor turns inactive and the later time at which it reappears as active. In the following examples we use

$$
\tilde{\rho}(\tau)=a^{2}(\tau-b) \exp [-a(\tau-b)]
$$

if $\tau \geqslant b$, and 0 otherwise. As $a$ increases, $\bar{\rho}$ becomes more and more peaked close to $\tau=b$. The expectation value of the waiting time $T_{w}$ is given by $T_{w}=b+2 / a$. Though completely ad hoc, this return probability density $\tilde{\rho}$ seems to define a fairly realistic model case. Since we do not have estimates for $a$ and $b$, we will freely vary them and study the consequences of this on the time-dependent ligand flux and active receptor population. We next present the numerical results for this case and shortly turn to a general derivation of the limiting values of $J(T) / J_{0}$ and $N(T) / N_{0}$.

We first consider the influence on the ligand current and the average active receptor population upon varying the variance in $\tilde{\rho}$, keeping $b=0$. Throughout, we keep $R / \alpha(0)=5$, which corresponds to typical values taken from [6]. In Figure 1 we plot $J(T) / J_{0}$ for sevcral $a$ values at two values of $\Gamma$. In Figure 2 the corresponding behavior of $N(T) / N_{0}$ is shown at the same parameter values. We notice that the small time behavior of $J(T) / J_{0}$ is essentially independent of these variations and closely resembles the behavior obtained for the case in which $\alpha$ is constant, which was treated in [6]. In addition, as a increases at constant $\Gamma, J(T) / J_{0}$ increases slightly, and this increase is more pronounced as $\Gamma$ is increased. However, $J(T) / J_{0}$ does not depend sensitively on variations of these parameters. Notice that as $a$ is decreased the stationary state sets in at a somewhat later time. The active receptor population $N(T) / N_{0}$ varies considerably as $a$ is varied; $N(T) / N_{0}$ decreases as $a$ is decreased, and again the decrease is more pronounced as $\Gamma$ is increased. It is remarkable that $N(T) / N_{0}$ shows a minimum for $T$ on the order of $T_{w}$.

In Figure $3 N(T) / N_{0}$ is plotted at $\Gamma=0.5$, showing the dependence of variations in $b$, keeping $a=1$. As $b$ is increased, $N(T) / N_{0}$ decreases, and the minimum in $N(T) / N_{0}$ occurs at a later time. Finally, the short-term behavior, up to the time where $N(T) / N_{0}$ is minimal, closely resembles the 


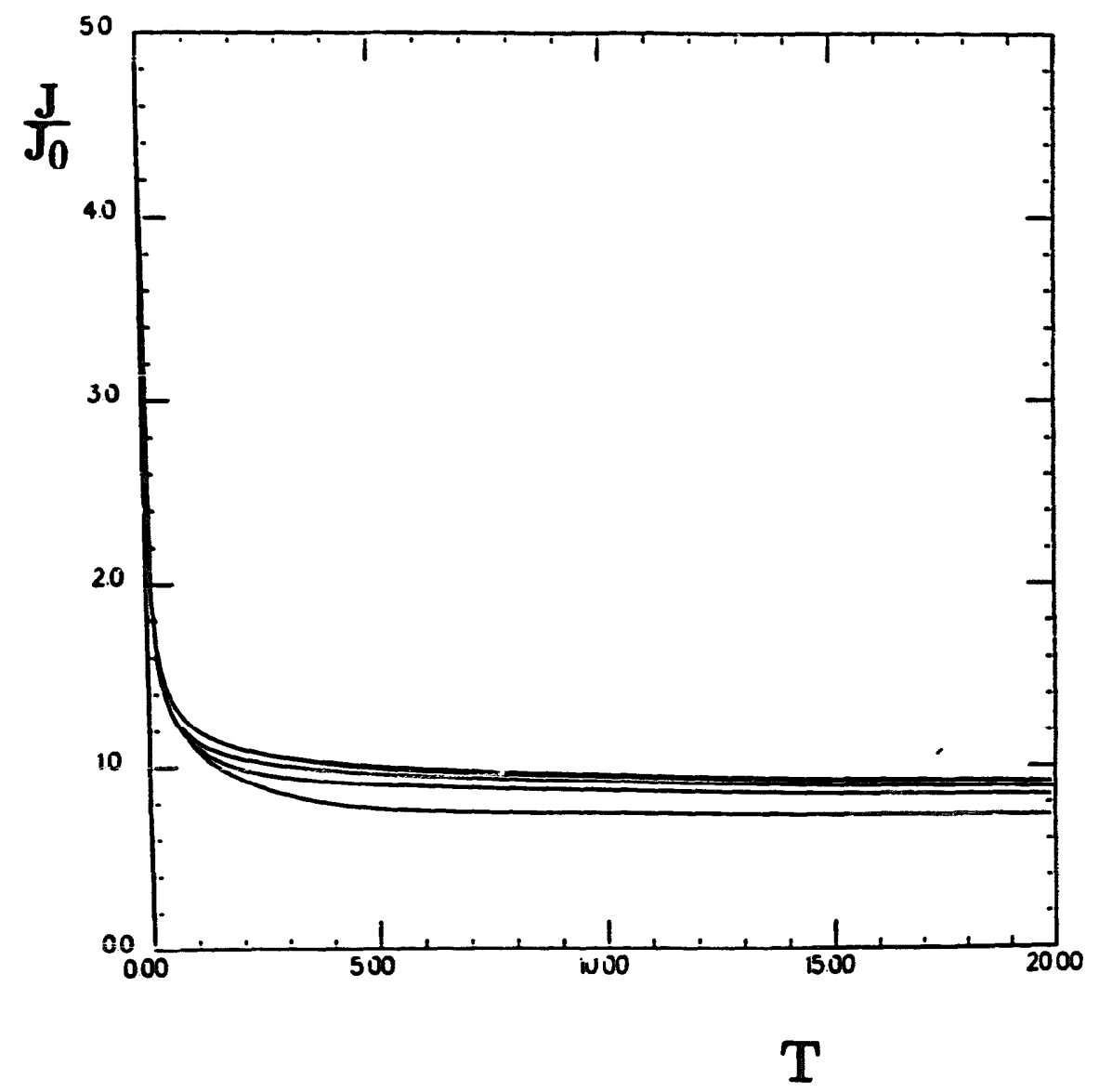

FIG. 1. A plot of the ligand current into the cell, $J(T) / J_{0}$, displaying the influence of varying the variance in the return probability at $b=0$. Notice that the ligand current does not depend sensitively on the parameter $a$. For the $a$ values used in the calculations, see Figure 2.

time dependence of $N(T) / N_{0}$ in the case $b \rightarrow \infty$, that, is the case in which there is complete inactivation of all receptors. This was treated separately in [7]. The behavior of $J(T) / J_{0}$ is qualitatively the same as in Figure 1, with $J(T) / J_{0}$ decreasing as $b$ increases.

We finally derive the limiting value of $N(T) / N_{0}$. As was shown in [4], the stationary-state ligand current into the cell can be written as

$$
J(\infty)=J_{0} \frac{N(\infty) / N_{0}}{N(\infty) / N_{0}+\alpha(0) / R},
$$

where $N(\infty)$ and $J(\infty)$ denote the limiting values of $N(T)$ and $J(T)$ 


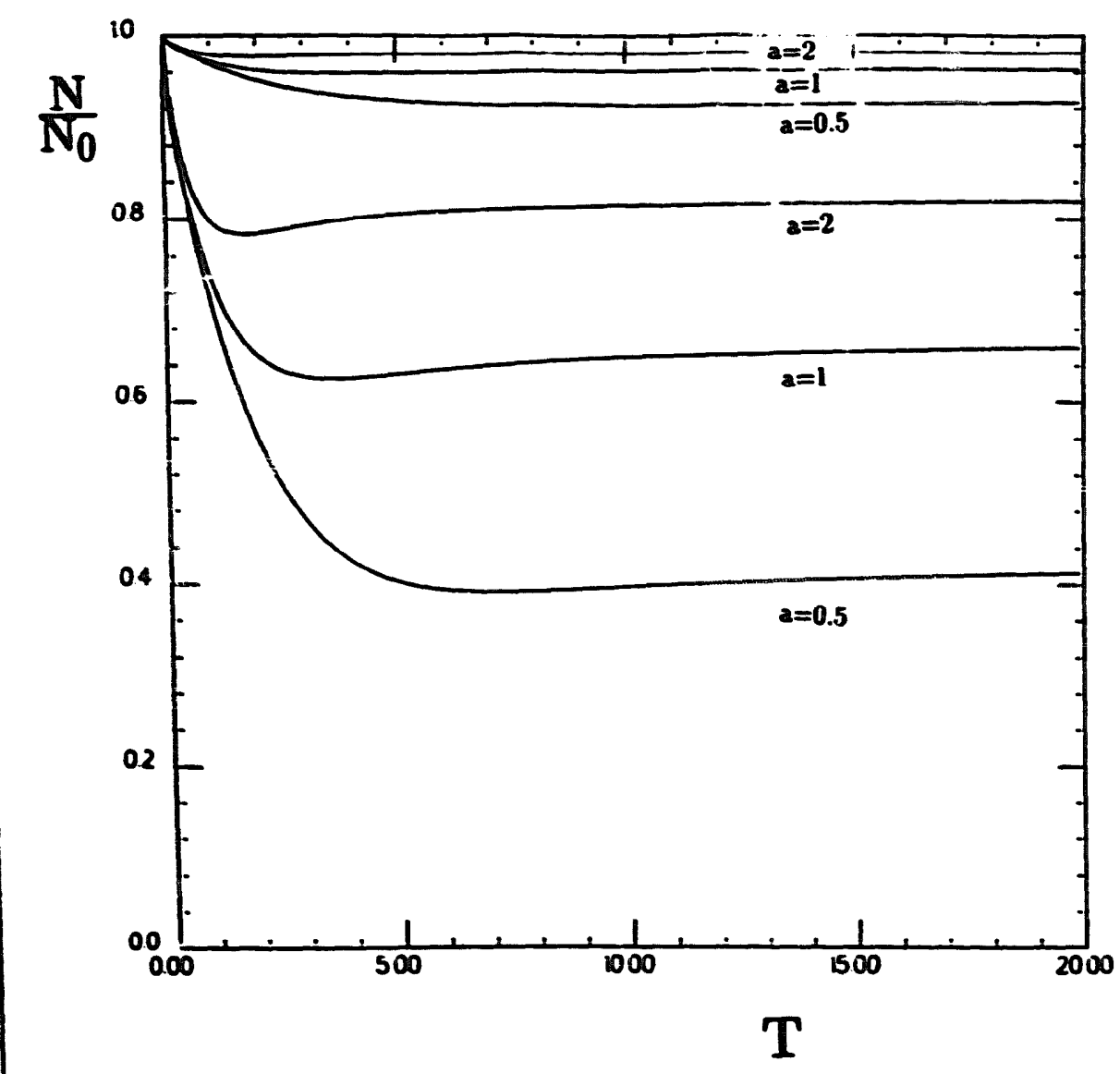

FIG. 2. The time dependence of the average active receptor population $N(T) / N_{0}$ showing the effect of varying the variance in the return probability at $b=0$. The upper three curves correspond to $r=0.1$ and the lower three to $\Gamma=1$.The values for $a$ used in the calculation are indicated rear the corresponding lines.

respectively, as $T \rightarrow \infty$. Hence, as $T \gg 1$ one has, to a good approximation, using (3.2) and (3.5),

$$
n(T) \approx 4 \pi R^{3} k c_{0} \frac{N(\infty) / N_{0}}{N(\infty) / N_{0}+\alpha(0) / R} \int_{0}^{T}\left[1-\int_{T}^{T} \tilde{\rho}\left(T^{\prime \prime}-T^{\prime}\right) d T^{\prime \prime}\right] d T^{\prime}
$$

This approximation becomes exact in the limit $T \rightarrow \infty$. Now, since

$$
\int_{0}^{T}\left[1-\int_{T^{\prime}}^{T} \tilde{\rho}\left(T^{\prime \prime}-T^{\prime}\right) d T^{\prime \prime}\right] d T^{\prime}=\int_{0}^{T} z \tilde{\rho}(z) \cdot d z+T \int_{T}^{\infty} \tilde{\rho}(z) d z,
$$




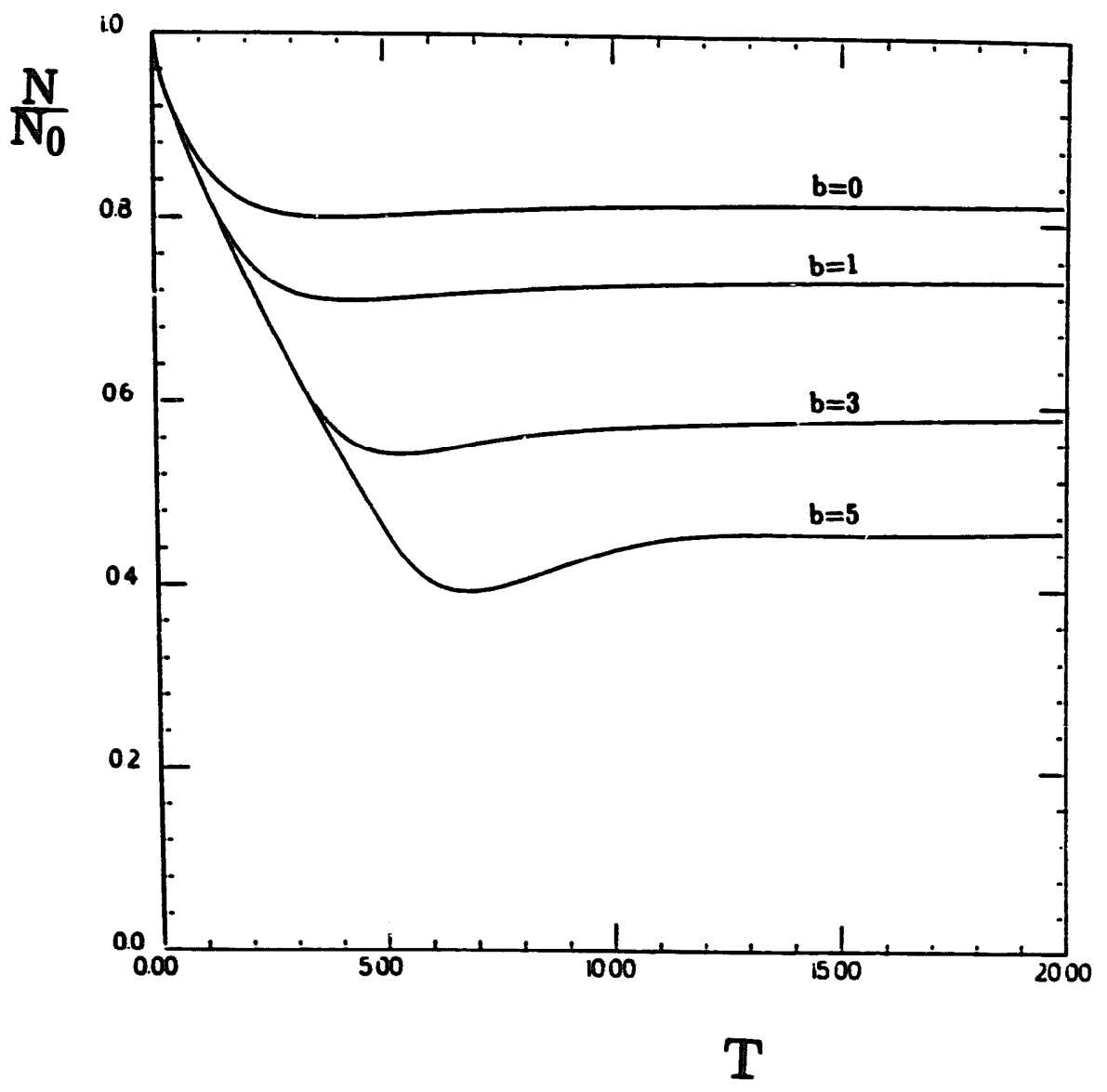

Fig. 3. The time dependence of the average active receptor population $N(T) / N_{0}$ showing the influence of variations in the delay time $b$ on the return probability at $a=1$. We used $\Gamma=0.5$, and the corresponding values for $b$ are indicated near the corresponding lines.

we find that $N(\infty) / N_{0}$ obeys the equation

$$
\frac{N(\infty)}{N_{0}}=1-\Gamma \frac{\alpha(0)}{R}\left(\frac{N(\infty) / N_{0}}{N(\infty) / N_{0}+\alpha(0) / R}\right) T_{w}
$$

provided $\tilde{\rho}$ decreases fast enough as $z$ becomes large, that is, $\tilde{\rho}<B z^{-(2+\epsilon)}$ with $B, \epsilon>0$ for $z \gg 1$. Here $T_{w}$ is the expectation value of the waiting time, which is finite given the above conditions. This quadratic equation can 


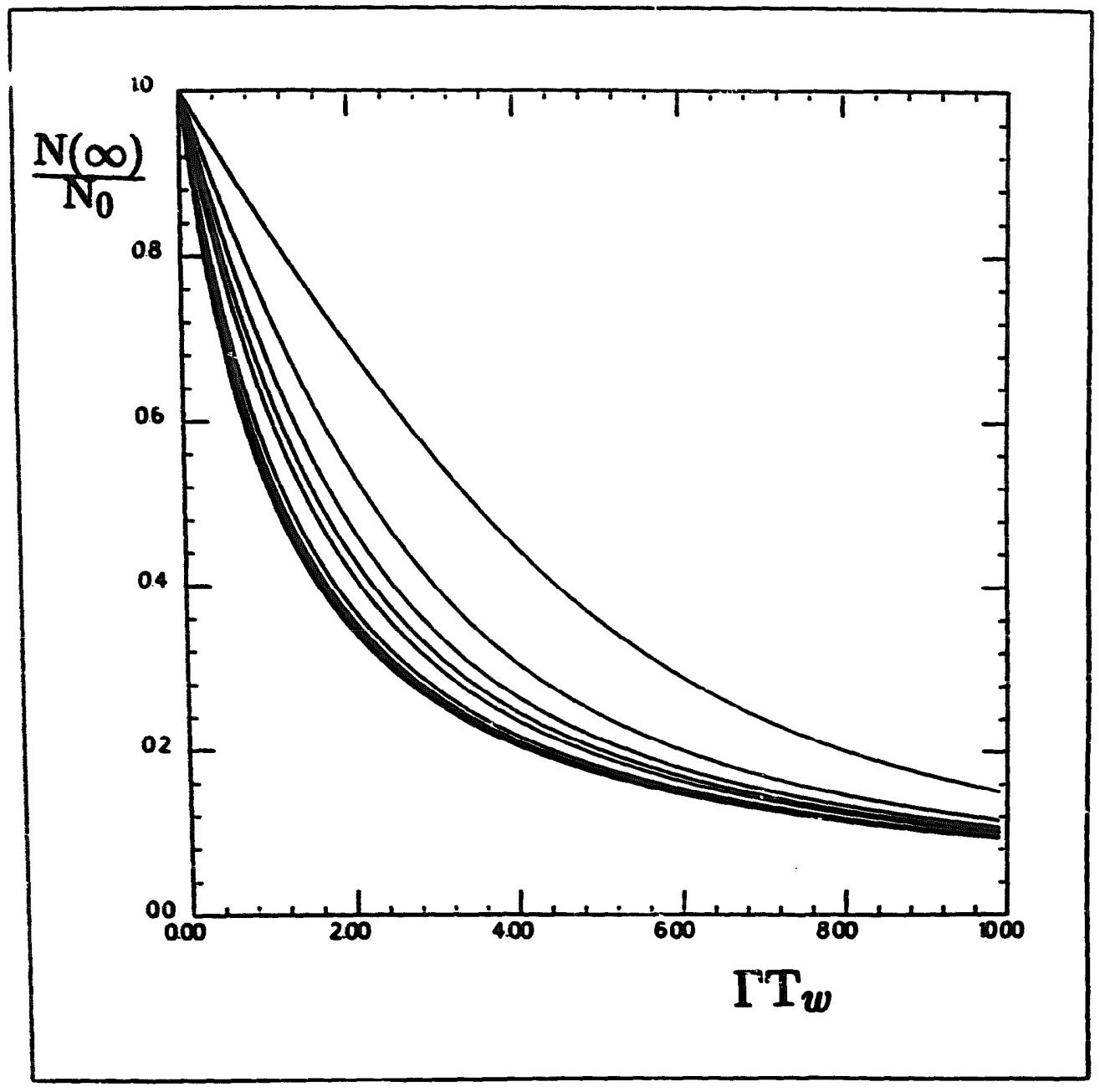

Frc. 4. The limiting values $N(\infty) / N_{0}$ as a function of $\Gamma T_{w}$ for various values of $\alpha(0) / R$. This upper five lines correspond to $\alpha(0) / R=0.2,0.4,0.6,0.8$, and 1.0 , respectively, from top to bottom, and the lower four with $\alpha(0) / R=2.0,3.0,4.0$, and 5.0, respectively, from top to bottom.

readily be solved to give

$$
\frac{N(\infty)}{N_{0}}=\frac{1}{2}\left\{\left(1-\frac{\alpha(0)}{R}\left(1+\Gamma T_{w}\right)\right)+\left[\left(\frac{\alpha(0)}{R}\left(1+\Gamma T_{w}\right)-1\right)^{2}+4 \frac{\alpha(0)}{R}\right]^{1 / 2}\right\}
$$

The other root of (4.5)must be disregarded since the average active receptor population satisfies $0 \leqslant N(\infty) \leqslant N_{0}$ for all parameter values. Notice that $N(\infty) / N_{0} \rightarrow 0$ as $T_{w} \rightarrow \infty$ and $N(\infty) / N_{0} \rightarrow 1$ as $T_{w} \rightarrow 0$, in accordance with 
what one may expect. In Figure 4 we plot $N(\infty) / N_{0}$ as a function of $\Gamma T_{w}$ for various values of $\alpha(0) / R$. We notice that $N(\infty) / N_{0}$ decreases as $\Gamma T_{w}$ increases. Comparison with the results obtained in Figures 2 and 3 for the case where $\tilde{\rho}$ is given by (4.1) shows that the limiting value $N(\infty) / N_{0}$ is reached only very slowly; typically the deviation of $N(T) / N_{0}$ from the corresponding limiting value is a few percent even for $T \approx 20$. Within this model, a cell can achieve complete adaptation, that is, $N(\infty) / N_{0} \approx$ constant for any $c_{0}$, by responding to slow changes in $c_{0}$ such that the product $\Gamma T_{w}$ remains constant. In view of (3.7), this implies that $T_{w}$ must be increased as $c_{0}$ decreases and vice versa.

\section{REFERENCES}

1 M. Abramowitz and I. A. Stegun, Handbook of Mathematical Functions, Dover, New York, 1964.

2 H. S. Carslaw and J. C. Jaeger, Conduction of Heat in Solids, Clarendon Press, Oxford, 1959.

3 P. J. Davis and P. Rabinowitz, Methods of Numerical Integration, Academic Press, London. 1975.

4 C. DeLisi and F. W. Wiegcl, Effect of nonspecific forces and finite receptor number on rate constants for ligand-cell bound-receptor interaction, Proc. Natl. Acad. Sci. U.S.A., 78:5569 (1981).

5 B. J. Geurts, Diffusion limited immunochemical sensing, Bull. Math. Biol. 51:359 (1989).

6 B. J. Geurts and F. W. Wiegel, Time dependent ligand current into a single cell performing chemoreception, Biophys. Chem. $28: 7$ (1987).

7 B. J. Geurts and F. W. Wiegel. Time dependent ligand current into a saturating cell performing chemoreception, Biophys. Chem. 31:317 (1988).

8 F. W. Wiegel, The physics of chemoreception, Phys. Rep. $95: 287$ (1983). 